\title{
Object permanence in dogs: Invisible displacement in a rotation task
}

\author{
Holly C. Miller, Cassie D. Gipson, Aubrey Vaughan, \\ RebeCCA RAYBURN-REEVES, AND ThOMAS R. ZENTALL \\ University of Kentucky, Lexington, Kentucky
}

\begin{abstract}
Dogs were tested for object permanence using an invisible displacement in which an object was hidden in one of two containers at either end of a beam and the beam was rotated. Consistent with earlier research, when the beam was rotated $180^{\circ}$, the dogs failed to find the object. However, when the beam was rotated only $90^{\circ}$, they were successful. Furthermore, when the dogs were led either $90^{\circ}$ or $180^{\circ}$ around the apparatus, they were also successful. In a control condition, when the dogs could not see the direction of the $90^{\circ}$ rotation, they failed to find the object. The results suggest that the $180^{\circ}$ rotation may produce an interfering context that can be reduced by rotating the apparatus only $90^{\circ}$ or by changing the dogs' perspective. Once the conflict is eliminated, dogs show evidence of object permanence that includes invisibly displaced objects.
\end{abstract}

By the age of 2 years, children develop the ability to search for objects that have disappeared from view. According to Piaget (1954), these search behaviors result from a developing knowledge about the nature of objects in the environment. Piaget called this ability object permanence and assessed it using both visible and invisible displacement tasks.

In a visible displacement task, an experimenter places a desired object inside one of several identical opaque containers (occluders), and the child is allowed to search for the displaced object. Children succeed at this task by about the age of 12 months (Piaget, 1954).

In the invisible displacement task, the object is placed in a container (the displacement device) that is then placed inside or behind an occluder. The object is then invisibly transferred from the displacement device into or behind the occluder. The displacement device (now empty) is shown to the child. Children successfully search inside the occluder that the displacement device last visited once they reach the age of about 2 years (Kramer, Hill, \& Cohen, 1975; Piaget, 1954). Piaget proposed that these children infer the location of the missing object because it is no longer in the displacement device.

Gorillas (Natale, Antinucci, Spinozzi, \& Potì, 1986), orangutans, chimpanzees (Call, 2001), Eurasian jays (Zucca, Milos, \& Vallortigara, 2007), and dogs (Gagnon \& Doré, 1992) have demonstrated the ability to search accurately for visibly displaced objects. However, the evidence for accurate searches by animals for invisibly displaced objects may be problematic. Gagnon and Doré (1993) reported that dogs search accurately for invisibly displaced objects when the object in a displacement device moves behind a screen and emerges empty. However, recent re- search has shown that cues provided by the displacement device and the location of the experimenter may account for the dogs' performance (Collier-Baker, Davis, \& Suddendorf, 2004; Fiset \& LeBlanc, 2007).

An alternative version of the invisible displacement task used by Doré, Fiset, Goulet, Dumas, and Gagnon (1996) involved the displacement of the occluder or screen after the object had been placed behind it. Doré et al. found that when the screen with the object was moved and was replaced with an empty screen, performance was very poor, but when the screen with the object was moved and no screen was moved to its previous location, performance was quite good.

Another version of the invisible displacement task used by Doré et al. (1996), which has been studied with children, involves a rotation apparatus on which three identical containers or occluders are evenly spaced (left, center, and right). The experimenter then hides a toy inside one of them and rotates the platform. Although 30-month-old children have been found to perform above chance (on average) when the object is rotated $180^{\circ}$ (Barth \& Call, 2006), if one discounts accurate searches directed at the center occluder (which remains in its original position when the platform is rotated), the children generally perform at levels below chance. Several great ape species perform similarly when tested with the $180^{\circ}$ rotation (Barth \& Call, 2006). Similarly, Fiset (2007) found that when two identical occluders are placed on either end of a rotating beam, and a hidden toy is displaced by $180^{\circ}$, dogs perform at or below chance (see also Bai \& Bertenthal, 1992, for similar results with children). That dogs fail to search accurately following a $180^{\circ}$ rotation is consistent with Watson et al. (2001), who concluded that dogs do not

T. R.Zentall, zentall@uky.edu 
show evidence for the logical abilities necessary to infer the invisible transfer of a hidden object.

However, it may be that the $180^{\circ}$ rotation task is exceptionally difficult for children, apes, and dogs because the occluder that contains the target object is replaced by an occluder that is empty. Additionally, the contextual or environmental cues present before and after the rotation remain the same. Thus, although the apparatus has been rotated, nothing appears to have changed.

In the present experiment, we asked whether dogs would be able to perform the invisible displacement rotation task if these interfering cues were eliminated by using a $90^{\circ}$ rotation rather than a $180^{\circ}$ rotation. With a $90^{\circ}$ rotation, the original position of the occluders would not compete with their final location.

A secondary purpose of the present experiment was to ask whether leading the dogs through either a $90^{\circ}$ rotation or a $180^{\circ}$ rotation (rather than rotating the apparatus) would also reduce the number of competing contextual cues and result in accurate performance because it would change the dogs' perspective (contextual cues would change).

\section{METHOD}

\section{Subjects}

Thirty-four dogs (Canis familiaris), 19 male and 15 female (ages ranging from 4 to 132 months, $M=55.9$ months), which belonged to private owners, were recruited. All dog owners were given a short questionnaire. Owners confirmed that their dogs matched several selection criteria. All dogs needed to be highly motivated by the opportunity to interact with the experimenters. Additionally, they needed to be highly motivated by food reinforcers. They also had to be comfortable with being handled when food reinforcers were nearby. Dogs were excluded if they had a history of aggressively guarding food resources. Finally, the owners had to be willing to deprive the dog of food for at least $4 \mathrm{~h}$ prior to participating. Of the dogs that participated in the experiment, 26 came from breeds classified by the American Kennel Club as herding dogs (11 Belgian tervuren, 5 Australian shepherds, 4 border collies, 2 Shetland sheepdogs, 2 Belgian sheepdogs, an Australian cattle dog, and a German shepherd), 3 were sporting dogs ( 2 Labrador retrievers and a Springer spaniel), 1 was a working dog (a Doberman pinscher), 1 was a terrier (a Scottish terrier), and 3 were mixed breeds. All of these dogs had been trained to sit and to walk next to their owners on command. Most of the dogs were naive to psychological experimentation. Four dogs had participated in previous, unrelated experiments.

\section{Apparatus}

A wooden beam $(1.83 \mathrm{~m}$ long $\times 14.0 \mathrm{~cm}$ wide $\times 3.8 \mathrm{~cm}$ thick $)$ was attached to a wooden base by a screw $(7.6 \mathrm{~cm}$ long) at its center. The screw rested inside a hole that was slightly larger in diameter and $2 \mathrm{~cm}$ deeper than the screw itself, so that the beam could easily rotate in the hole. A $1.83-\mathrm{m}$ length of transparent fishing line $(5.5 \mathrm{~kg}, .30 \mathrm{~mm}$ in diameter) was attached to each end of the beam to allow the beam to be rotated from a distance. Two identical opaque occluders were attached to the beam, one on each end. The occluders (containers $25.4 \mathrm{~cm}$ wide $\times 30 \mathrm{~cm}$ high $\times 20 \mathrm{~cm}$ deep) were each fitted with false bottoms. Each false bottom $(20 \mathrm{~cm}$ wide $\times 10 \mathrm{~cm}$ high $\times 14 \mathrm{~cm}$ deep) contained 1 cup of dog treats that was covered tightly with a plastic lid that was perforated to allow the odor of the treats to escape. The treats were the same as those that were used to bait the occluders and were replenished before each dog was tested. The treats (Pet Botanics semimoist Chicken \& Brown Rice dinner dog food) were cut into portions of about $2 \mathrm{~g}$. Each false bottom also contained one of three identical plastic bowls $(15 \mathrm{~cm}$ diameter $\times 7.5 \mathrm{~cm}$ deep). One of the bowls served as the target object for all dogs except 1 . This 1 dog was motivated to work for food, but was even more motivated to interact with his favorite toy. All of the conditions remained the same for this dog, except that a toy was used as his target object. For a control condition in which visual cues were eliminated, an opaque barrier ( $1.40 \mathrm{~m}$ wide $\times 1.22 \mathrm{~m}$ tall) was constructed from a metal frame, on which an opaque cloth was draped.

All of the testing took place in locations that were familiar to the dog. The most common locations were inside a house $(n=15)$ or in a yard $(n=14)$. Some dogs were tested at the location of their dog obedience classes $(n=5)$. All of the locations were large enough to accommodate the full rotation of the apparatus or movement of the dog with its owner and the experimenter. A digital video camera (Sony Model No. DCR-HC26) was used to record the dogs' choices.

\section{Procedure}

Both the visible and invisible displacement tests were administered in a single session, lasting approximately $20 \mathrm{~min}$. Dogs were tested individually, with their owners present. The owners restrained the dogs when necessary. The experimenter spent the first 5 min of the session familiarizing the dog with the apparatus and the owner with the procedure. Following this familiarization period, the experimenter trained the dog to associate a bowl with food. This was done by placing a treat inside a bowl and offering the bowl to the dog. Once the dog had eaten out of each of the three bowls, one of the bowls was placed inside each of the false bottoms. The third was baited and offered to the dog once more. After the training trials, the experimenter allowed the unrestrained dog to follow her as she held the bowl. The bowl was not obscured from the dog's view (the dog was close enough to physically follow the bowl at all times) as it was placed inside an occluder. The dog was allowed to immediately consume the food. Once the left occluder had been baited, the experimenter walked directly over to the right container (while the dog followed) and baited it. The order was counterbalanced. The purpose of this pretraining was to allow the subjects to experience the hiding potential of the occluders, as well as to ensure that the dogs would put their heads inside the occluders to reach the target object.

In all of the tests, the dogs sat approximately $2 \mathrm{~m}$ away from the center of the apparatus (a distance determined by the size of the dog; each dog was far enough away from the apparatus that it could not see inside the occluder when it was directly in front of the dog). The owners stood to the right of the dog and manually restrained the dog by the collar in a position that was equidistant from each occluder to prevent the dog from initiating any preemptory movement forward. To reduce the possibility of inadvertent cues, the owner faced away from the apparatus whenever possible. If this was not possible (e.g., in the conditions in which the dog was moved), the owner was instructed to look at a point on the wall above the apparatus and to avoid interacting with the dog during the trial. A phrase such as "Okay!" or "Find it" was arranged with the owner to serve as a signal to release the dog. When giving this signal, the experimenter was careful to avoid giving any cues about the object's location.

\section{Visible Displacement Tests}

All visible displacement tests began with the experimenter standing $1.0 \mathrm{~m}$ behind the center of the apparatus and approximately $3 \mathrm{~m}$ in front of the dog. The beam was placed perpendicular to the dog, so that one occluder was to the right of the dog and the other occluder was to the left of the dog (see Figure 1A). The experimenter placed a treat inside the target bowl and attracted the dog's attention by saying a phrase such as "Cookie!" or "Puppy, puppy, puppy!" Once the dog was visually attending, the experimenter proceeded to walk to the left or right occluder (each occluder and location was randomly assigned) and to place the bowl inside the occluder and on top of the false bottom. The experimenter then quietly backed away from the occluder, assumed a neutral position that was equidistant from each occluder, and cued the release of the dog. The dog was then allowed to approach either occluder. Any physical contact with an occluder, or visual in- 
A

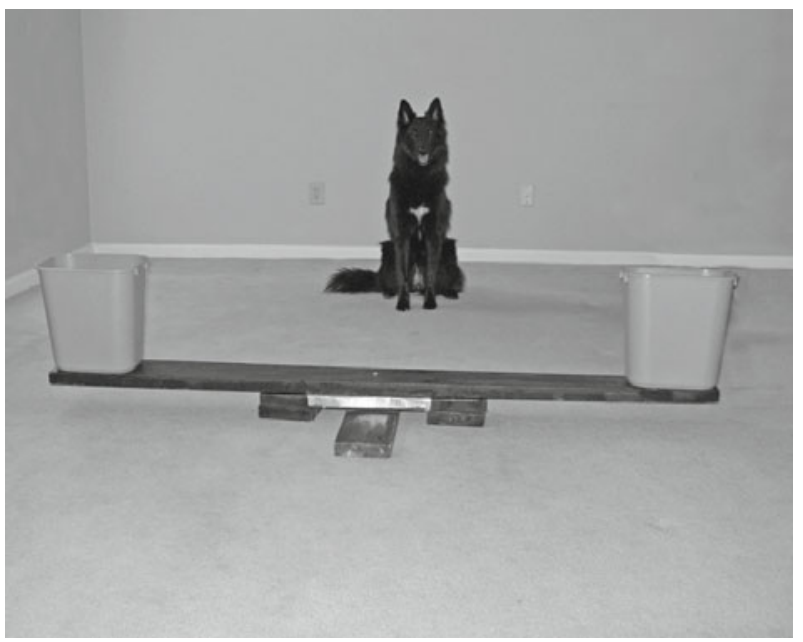

B

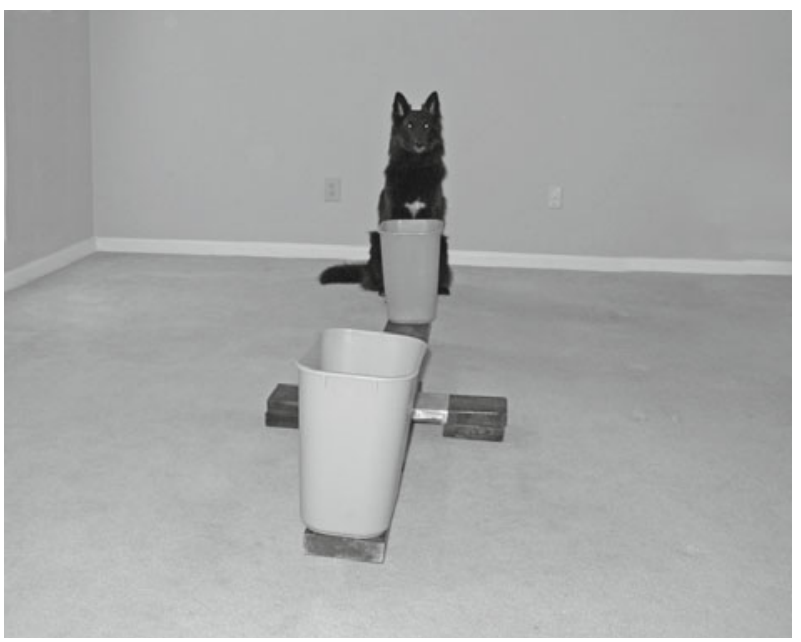

Figure 1. (A) Apparatus and dog in the visible displacement position and in the starting positions for the $180^{\circ}$ invisible displacement conditions, in which the apparatus was rotated or the dog was led. (B) Apparatus and dog in the visible displacement position (for the last two trials) and in the starting positions for the $90^{\circ}$ invisible displacement conditions, in which the apparatus was rotated or the dog was led.

spection of its contents, was considered a choice. Most dogs retrieved the food reward following a correct choice by physically inserting their head inside the occluder. All of the dogs were rewarded with additional verbal praise for a correct choice. If the dogs did not choose correctly, the experimenter used the phrase "Too bad" before removing the bowl from the correct occluder. The owner then retrieved the dog. There were eight visible displacement trials. The last two visible displacement trials began with the beam placed in line with the dog (see Figure 1B). The occluder nearest the dog was baited, and each occluder was baited once in this way. The farther occluder was not used because it was partially obscured from view by the nearer occluder.

\section{Invisible Displacement Tests}

There were five different invisible displacement groups. A schematic diagram of the five invisible displacement conditions appears in Figure 2. The first group $(n=17)$ observed a $180^{\circ}$ rotation of the apparatus. The second group $(n=13)$ observed a $90^{\circ}$ rotation of the apparatus. A subset of the 13 dogs $(n=6)$ was retested (the third group) with a barrier placed between the dog and the apparatus in such a way that the dog could not see the $90^{\circ}$ rotation of the beam, to control for nonvisual cues. Each dog in the fourth group $(n=7)$ observed a visible displacement before it was 1 ed $90^{\circ}$ from the baited occluder. Each dog in the fifth group $(n=7)$ observed a visible displacement before it was led $180^{\circ}$ from the baited occluder. For all of the dogs, the final location of the baited occluder was to the left or right (equally often). The same side was not baited more than twice in succession. All 12 invisible displacement test trials began with the experimenter standing about $1 \mathrm{~m}$ behind the center of the apparatus and approximately $3 \mathrm{~m}$ in front of the dog. In the $90^{\circ}$ conditions, the experimenter stood directly behind the far occluder and the dog was positioned approximately $1 \mathrm{~m}$ away from the near occluder. If the dog moved away from the apparatus rather than toward it, the trial was aborted and then was repeated. A total of 6 trials were repeated for this reason.

$180^{\circ}$ rotation of the apparatus. In this condition, the beam was placed perpendicular to the dog so that the occluders were equidistant from the dog (see Figure 1A). The experimenter placed a treat in the bowl before attracting the dog's attention. Once the dog was visually attending, the experimenter walked toward an occluder (randomly assigned) and placed the bowl inside. The experimenter then collected the nylon line attached to that end of the beam and then collected the nylon line attached to the other end. The experimenter used these lines to rotate the beam $180^{\circ}$ (the direction was randomly assigned). Once the beam was rotated, the experimenter assumed a neutral position behind the center of the apparatus and then cued the release of the dog.

$90^{\circ}$ rotation of the apparatus. The beam was placed in line with the dog so that one occluder was directly in front of the dog (see Figure 1B). The experimenter placed a treat in the bowl before attracting the dog's attention. Once the dog was visually attending, the experimenter walked toward the dog on, for instance, the right side of the beam and placed the bowl inside the occluder. The experimenter then collected the nylon line attached to that end of the beam and then backed away to collect the nylon line attached to the other end. While standing behind the far occluder, the experimenter used the lines to rotate the beam $90^{\circ}$ (see Figure 1A; the occluder and direction of rotation were randomly assigned). Once the beam was rotated, the experimenter assumed a neutral position behind the center of the apparatus and then cued the release of the dog.

Control condition: $90^{\circ}$ rotation of the apparatus with visual barrier. The procedure was identical to the $90^{\circ}$ rotation of the apparatus (including a repeat of the visible displacement test), except that a visual barrier was used to block the dog's view of the rotation during the invisible displacement tests. This condition served as a control to assess the possibility that nonvisual cues could be used to detect the location of the treat. Once the occluder had been baited, an opaque screen was placed between the dog and the apparatus by a second experimenter. The screen, which served to block the dog's view of the rotation but not of the experimenter, remained in place while the apparatus was rotated $90^{\circ}$. Once the rotation had been completed and the experimenter had assumed a neutral position, the screen was removed. Then, the experimenter cued the release of the dog. Three trials were repeated for 1 dog because the experimenter noticed that it had moved so it could see around the barrier during the rotation.

Leading the $\operatorname{dog} 90^{\circ}$ relative to the apparatus. This procedure began in the same way as the other $90^{\circ}$ conditions (see Figure 1B), except that once the front occluder was baited, the experimenter backed away to the center of the apparatus. The experimenter then moved perpendicular (left or right) from the apparatus (direction was randomly assigned) before assuming a neutral position facing the center of the apparatus approximately $1.0 \mathrm{~m}$ away. The owner then led the dog in the heel position (the dog's withers were aligned with the owner's heels) either to the right or to the left in a $90^{\circ}$ arc 

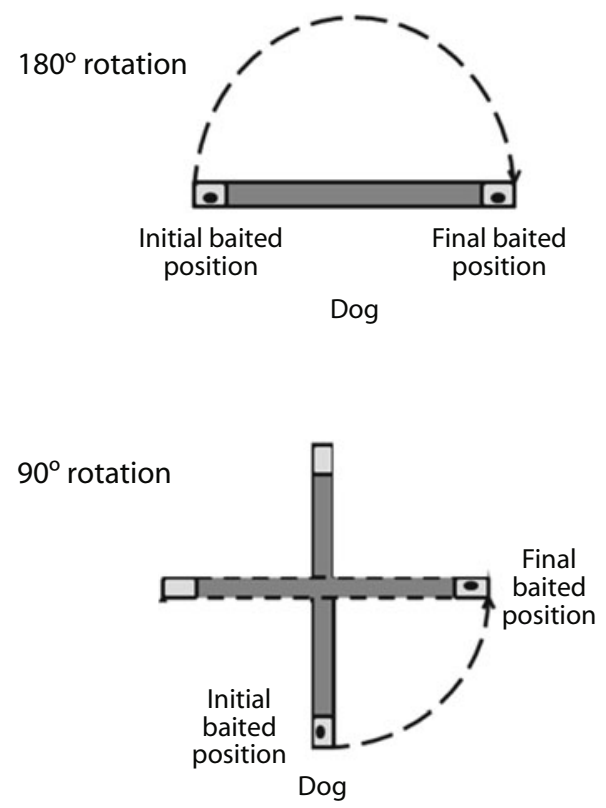

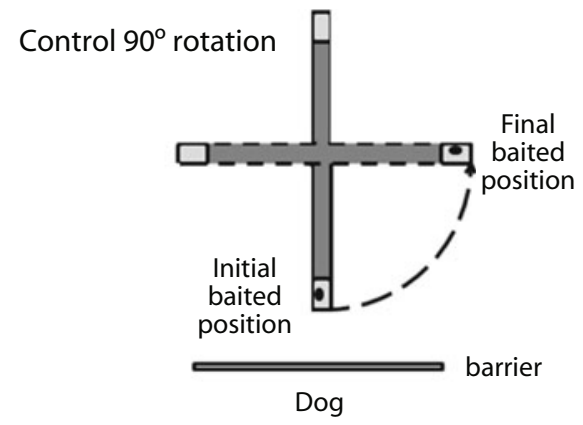

Dog led $90^{\circ}$

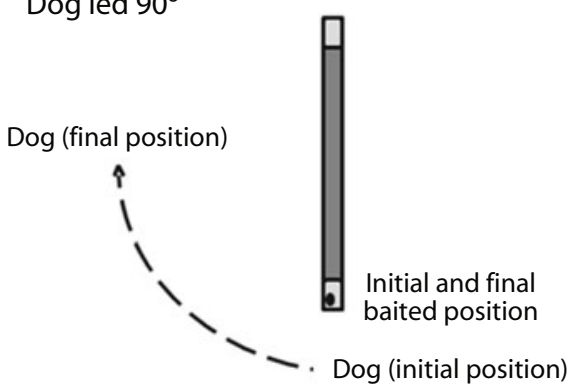

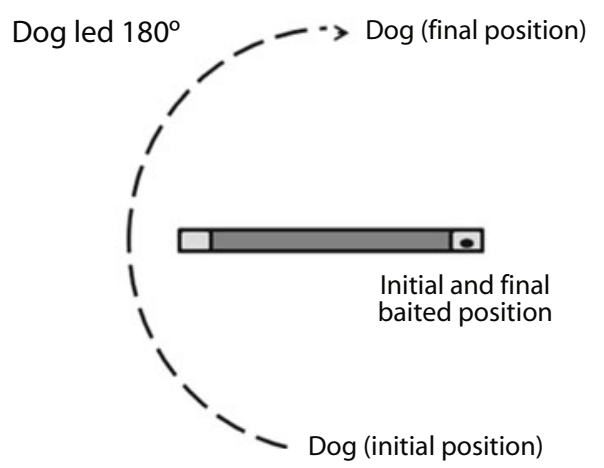

Figure 2. Schematic diagram of the five conditions of invisible displacement. $180^{\circ}$ rotation: A treat was placed in either occluder, and the apparatus was rotated $180^{\circ} .90^{\circ}$ rotation: A treat was placed in the closest occluder, and the apparatus was rotated $90^{\circ}$. Control $90^{\circ}$ rotation: A treat was placed in the closest occluder and the apparatus was rotated $90^{\circ}$, but a barrier was placed in front of the dog so that its view of the apparatus was obscured. Dog led $90^{\circ}$ : A treat was placed in the closest occluder, and the dog was led $90^{\circ}$ in either direction. Dog led $180^{\circ}$ : A treat was placed in either occluder, and the dog was led $180^{\circ}$.

away from the experimenter, in such a way that it maintained the 3-m distance from the occluder (to prevent the dog from seeing inside). Once the dog reached a position equidistant from each occluder and directly across from the experimenter, the owner was cued to release the dog. The location of the dog relative to the apparatus was counterbalanced; the first six trials began with the dog on one side of the apparatus, and the remaining six trials began with the dog on the opposite side.

Leading the $\operatorname{dog} 180^{\circ}$ relative to the apparatus. In this condition, the beam was placed perpendicular to the dog so that each occluder was equidistant from the dog (see Figure 1A). The experimenter baited the bowl, walked to an occluder (randomly assigned), and placed the bowl inside. The experimenter then moved $2.5 \mathrm{~m}$ behind the center of the apparatus and assumed a neutral position. The owner then walked the dog in a $180^{\circ}$ arc (from the left or the right, randomly assigned) until the dog was directly in front of the experi- menter and was facing the center of the apparatus. Once the dog was in position, the experimenter cued the release of the dog.

\section{RESULTS}

\section{Visible Displacement}

Mean visible displacement search accuracy for the dogs that would later serve in the five test conditions appears in Figure 3. On the eight visible displacement trials, the dogs searched accurately for the visibly displaced object $94 \%$ of the time (trials correct ranged from 5 to $8, M=$ 7.5). Accuracy was significantly different from chance $[t(33)=27.54, p<.01, d=9.59]$. A one-way ANOVA on the data from the four experimental groups with group 


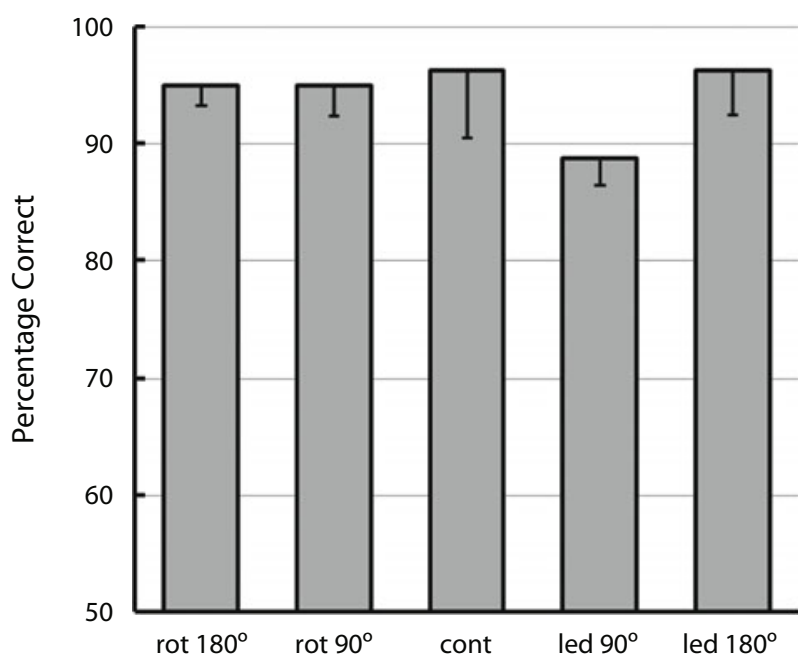

Figure 3. Mean accuracy on visible displacement tests that preceded the invisible displacement tests for each invisible displacement condition. Error bars represent one standard error of the mean. The conditions that followed the visible displacement tests were apparatus rotated $180^{\circ}\left(\right.$ rot $\left.180^{\circ}\right)$, apparatus rotated $90^{\circ}$ (rot $\left.90^{\circ}\right)$, barrier control (cont), dog led $90^{\circ}\left(\operatorname{led} 90^{\circ}\right)$, and $\operatorname{dog} \operatorname{led} 180^{\circ}$ (led $\left.180^{\circ}\right)$.

as a factor indicated that the difference among the conditions was not statistically significant $[F(3,30)<1]$.

\section{Invisible Displacement}

Mean invisible displacement search accuracy for the five test conditions appears in Figure 4. The average search accuracy for the dogs that observed a $180^{\circ}$ rotation of the apparatus was $34 \%$ (trials correct ranged from 1 to $6, M=4.1$ ). When the apparatus was rotated $90^{\circ}$, the dogs searched the correct location $82 \%$ of the time (trials correct ranged from 6 to $12, M=9.8$ ). When visual cues were eliminated, average accuracy dropped to approximately chance $(53 \%$; trials correct ranged from 6 to 7 , $M=6.3)$. When the dogs were led $90^{\circ}$ around the apparatus, they searched accurately $(82 \%$; trials correct ranged from 8 to $12, M=9.9$ ), and they also performed at a high level $(81 \%)$ when they were led $180^{\circ}$ around the apparatus (trials correct ranged from 6 to $12, M=9.7$ ).

The dogs that observed a $180^{\circ}$ rotation of the apparatus performed significantly below chance $[t(6)=2.64$, $p=.03, d=2.16]$. However, when dogs observed a $90^{\circ}$ rotation of the apparatus, they performed significantly above chance $[t(12)=8.55, p<.01, d=4.94]$. When visual cues were eliminated from the $90^{\circ}$ rotation, performance dropped to near chance $[t(5)=1.58, p=.17]$, but when the dogs were led $90^{\circ}$ and $180^{\circ}$ around the apparatus they searched significantly more accurately than chance $[t(6)=7.59, p<.01, d=6.20$, and $t(6)=4.16, p<.01$, $d=3.40$, respectively].

A correlated $t$ test was conducted in order to compare accuracy when the apparatus was rotated $90^{\circ}$ with and without visual cues. Dogs' accuracy was significantly lower when visual cues were eliminated $[t(5)=3.11, p=$ $.02, d=2.78]$.
A $2 \times 2$ ANOVA was conducted with degree of rotation and whether the apparatus was rotated or the dog was led as factors. The dogs in the $90^{\circ}$ conditions searched more accurately than those in the $180^{\circ}$ conditions $[F(1,30)=$ 21.34, $p<.01, d=1.69]$. Also, the dogs that were led around the apparatus searched more accurately than those that observed the apparatus rotate $[F(1,30)=10.21, p<$ $.01, d=1.17]$. Finally, there was a significant interaction between the degree of rotation and apparatus rotation versus dogs being led $[F(1,30)=23.95, p<.01, d=$ $1.79]$, which resulted from the high level of performance when the dog was led $180^{\circ}$ around the apparatus. All of the effects in this analysis were produced by the poor performance by dogs that observed the $180^{\circ}$ rotation.

\section{DISCUSSION}

Fiset (2007) found that when two identical occluders are placed on either end of a rotating beam, dogs perform at or below chance $(50 \%)$ when searching for a hidden toy that was displaced $180^{\circ}$. The results of our group that observed the $180^{\circ}$ rotation of the apparatus confirmed this failure. We argued that the poor performance on the rotation task was likely due to conflicting contextual cues. The apparatus appeared the same before and after the rotation. Once these conflicting cues were eliminated (by rotating the apparatus only $90^{\circ}$ ), the dogs searched accurately for the invisibly displaced object. We also found that when the dogs were led around the apparatus either $90^{\circ}$ or $180^{\circ}$, they also searched accurately. Dogs in a control condition, which were not able to see the $90^{\circ}$ rotation, failed to search accurately.

Gagnon and Doré (1992) found that performance on the traditional invisible displacement test was better when it was preceded by the visible test. For this reason, we used that order of testing in the present study. It may be that

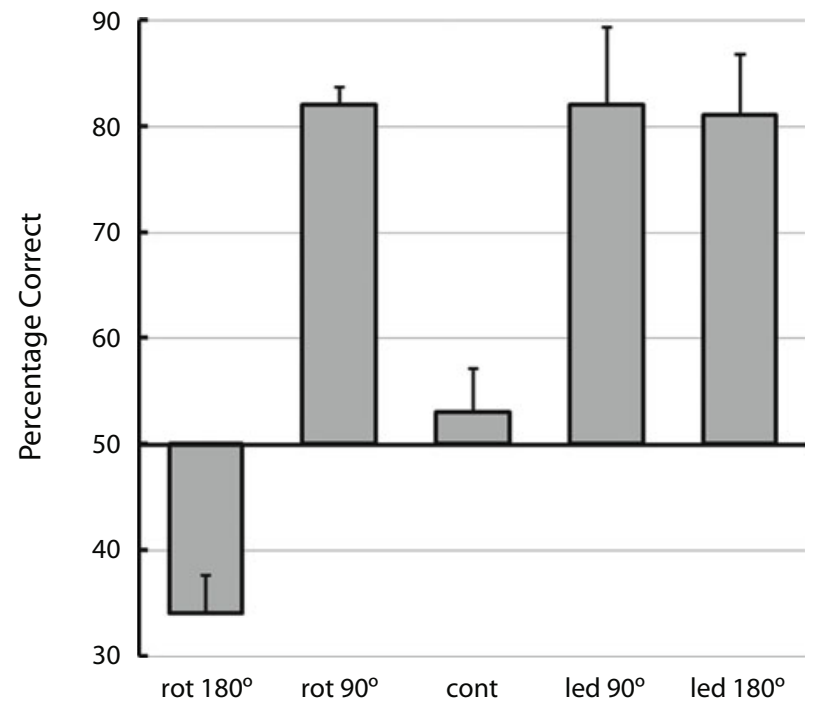

Figure 4. Mean accuracy on invisible displacement tests. Error bars represent one standard error of the mean. rot $180^{\circ}$; apparatus rotated $180^{\circ}$; rot $90^{\circ}$, apparatus rotated $90^{\circ}$; cont, barrier control; led $90^{\circ}$, dog led $90^{\circ}$; led $180^{\circ}$, dog led $180^{\circ}$. 
prior visible displacement testing affected the dogs' incentive motivation or increased attention to the occluders, but it does not account for the fact that the dogs were able to track the $90^{\circ}$ rotation but not the $180^{\circ}$ rotation.

Piaget (1954) believed that successful performance on the object permanence task suggested the ability to represent an object that is not present. But successful performance of the rotational version of the invisible displacement task may be explained in terms of a higher order conditioning process. Initially, the dogs associated the bowl with food. When the bowl was then placed inside the occluder, the occluder was associated with food by way of the bowl (higher order conditioning). If the dogs visually tracked and then approached this higher order conditioned stimulus, they would search in the appropriate occluder.

The results of the manipulation in which the dog was led around the apparatus offer additional support for the hypothesis that their failure to search accurately in the $180^{\circ}$ rotation condition can be attributed to conflicting contextual cues. Not only did the dogs perform well in the condition in which they were led $90^{\circ}$ around the apparatus, but they also performed well in the condition in which they were led $180^{\circ}$ around the apparatus. What differentiated the two $180^{\circ}$ conditions was the change in context experienced by the dogs that were led around the apparatus but not by the dogs for which the apparatus was rotated.

The fact that the present experiment was conducted in the presence of a human experimenter leaves open the possibility that inadvertent social cues were detected by the dogs. Although the experimenter went to great lengths to avoid providing such cues, the best evidence that social cues did not play a significant role in this experiment is the performance of dogs in the $180^{\circ}$ rotation and control conditions. In both conditions, had inadvertent social cues been a factor, the dogs would have performed more accurately. The same can be said for the possibility that differential olfactory cues guided the dogs' search behavior. Had differential odor cues been present, dogs in the $180^{\circ}$ rotation and control conditions would have searched more accurately.

In the present experiment, the last two trials of the visible displacement test began with one occluder in front of the dog. This was done to familiarize the dog with the different locations that the two occluders would be in during the invisible displacement test. To determine whether these trials contributed to accurate invisible displacement performance, we recently tested 6 dogs in the $90^{\circ}$ rotation condition without the last two visible displacement trials. When these dogs were tested with invisible displacement trials, they chose correctly $91.7 \%$ of the time.

The results of the present experiment demonstrate that when interfering contextual cues are eliminated, dogs search accurately for an invisibly displaced object without the need for explicit training. Whether this form of invisible displacement is interpreted as evidence for the representation of a hidden object by dogs or for a form of higher order conditioning, it does identify the conditions under which such accurate search can be found.

\section{AUTHOR NOTE}

We thank Kristina Pattison for her help in conducting this study, Libbye Miller for her photographic help, and both of them for their help in recruiting subjects. We also thank the many dog owners who participated in this study; without their help and cooperation, this research would not have been possible. Preparation of this article was supported by National Institute of Mental Health Grant MH-063726 to T.R.Z. Correspondence concerning this article should be addressed to T. R. Zentall, Department of Psychology, University of Kentucky, Lexington, KY 40506 (e-mail: zentall@uky.edu).

\section{REFERENCES}

BAi, D. L., \& Bertenthal, B. I. (1992). Locomotor status and the development of spatial search skills. Child Development, 63, 215-226.

BARTH, J., \& CALL, J. (2006). Tracking the displacement of objects: A series of tasks with great apes (Pan troglodytes, Pan paniscus, Gorilla gorilla, and Pongo pygmaeus) and young children (Homo sapiens). Journal of Experimental Psychology: Animal Behavior Processes, 32, 239-252.

CALl, J. (2001). Object permanence in orangutans (Pongo pygmaeus), chimpanzees (Pan troglodytes), and children (Homo sapiens). Journal of Comparative Psychology, 115, 159-171.

Collier-Baker, E., Davis, J. M., \& Suddendorf, T. (2004). Do dogs (Canis familiaris) understand invisible displacement? Journal of Comparative Psychology, 118, 421-433.

Doré, F. Y., Fiset, S., Goulet, S., Dumas, M.-C., \& Gagnon, S. (1996). Search behavior in cats and dogs: Interspecific differences in working memory and spatial cognition. Animal Learning \& Behavior, 24, 142-149.

FISET, S. (2007, March). Spatial rotation of hidden objects in domestic dogs. Paper presented at the meeting of the International Conference on Comparative Cognition, Melbourne, FL.

Fiset, S., \& LeBlanc, V. (2007). Invisible displacement understanding in domestic dogs (Canis familiaris): The role of visual cues in search behavior. Animal Cognition, 10, 211-224.

GAGNON, S., \& DORÉ, F. Y. (1992). Search behavior in various breeds of adult dogs (Canis familiaris): Object permanence and olfactory cues. Journal of Comparative Psychology, 106, 58-68.

GaGnon, S., \& Doré, F. Y. (1993). Search behavior of dogs (Canis familiaris) in invisible displacement problems. Learning \& Behavior, 21, 246-254

Kramer, J. A., Hill, K. T., \& Cohen, L. B. (1975). Infants' development of object permanence: A refined methodology and new evidence for Piaget's hypothesized ordinality. Child Development, 46, 149-155.

Natale, F., Antinucci, F., Spinozzi, F., \& Potì, P. (1986). Stage 6 object permanence in nonhuman primate cognition: A comparison between gorilla (Gorilla gorilla) and Japanese macaque (Macaca fuscata). Journal of Comparative Psychology, 100, 335-339.

Piaget, J. (1954). The construction of reality in the child. New York: Basic Books.

Watson, J. S., Gergely, G., Csanyi, V., Topal., J., Gacsi, M., \& SARKOZI, Z. (2001). Distinguishing logic from association in the solution of an invisible displacement task by children (Homo sapiens) and dogs (Canis familiaris): Using negation of disjunction. Journal of Comparative Psychology, 115, 219-226.

Zucca, P., Milos, N., \& Vallortigara, G. (2007). Piagetian object permanence and its development in Eurasian jays (Garrulus glandarius). Animal Cognition, 10, 243-258.

(Manuscript received May 7, 2008; revision accepted for publication July 19, 2008.) 